\title{
COVID-19: The Impact in Oncology Care
}

\author{
Upasana Ray ${ }^{1} \cdot$ Faisal Aziz ${ }^{2} \cdot$ Abhishek Shankar $^{3} \cdot$ Aalekhya Sharma Biswas $^{4} \cdot$ Abhijit Chakraborty $^{4}$ (D)
}

Accepted: 13 October 2020 / Published online: 23 October 2020

(C) Springer Nature Switzerland AG 2020

\begin{abstract}
The COVID-19 pandemic has imposed a critical challenge to the current oncology care and practices including late diagnoses, delayed anti-cancer treatment, and static clinical trials. With the increasing risk of cancer patients acquiring infection during receiving the essential care, the debate ensues on how to balance the risk factors and benefits out of the oncologic emergencies in cancer patients. In this review article, we have focused on the current global re-organization of the integrity and effectiveness of the treatment modalities depending on the patient and cancer-specific urgencies while minimizing exposure to the infection. In this review, we addressed how the worldwide oncology community is united to share therapy schemes and the best possible guidelines to help cancer patients, and to strategize and execute therapy/trial protocols. This review provides collective knowledge on the current re-structuring of the general framework that prioritizes cancer care with the available exploitation of the reduced resources and most importantly the unparalleled levels of companionship as a large health care community towards the need to offer the best possible care to the patients.
\end{abstract}

Keywords SARS-CoV-2 $\cdot$ Cancer $\cdot$ SARS $\cdot$ MERS

\section{Introduction}

In December 2019, a distinctive coronavirus (CoV) was determined to be responsible for an outbreak of potentially fatal atypical pneumonia, ultimately defined as coronavirus disease

\section{Highlights}

- The biology behind the SARS-CoV-2 infection and its difference from SARS and MERS.

- We have discussed the current statistics on the risk of COVID-19 infection and the high susceptibility of the cancer patients towards being infected.

- We have addressed the treatment prioritization with the balancing of risk and benefits depending on the patient and cancer-specific emergencies.

-We provided important perspectives in a nutshell from oncology-emergencies, values, and strategic maintenance frameworks to highlight the present scenario in the field of clinical oncology.

This article is part of the Topical Collection on Covid-19

Abhijit Chakraborty

abhijit.chakraborty@bcm.edu

1 Mayo Clinic, Rochester, MN, USA

2 The Hormel Institute, University of Minnesota, Austin, MN, USA

3 Lady Hardinge Medical College and Associated Hospitals, Delhi, India

4 Baylor College of Medicine, Houston, TX, USA
19 (COVID-19), in Wuhan, Hubei province, China [1]. Early cases of Severe acute respiratory syndrome coronavirus 2 (SARS-CoV-2) were identified in people who visited or worked in the Huanan Wholesale Seafood Market in Wuhan. From Wuhan, China SARS-CoV-2 is dramatically spreading in several months all over the world. The disease related to SARS-CoV-2 is called coronavirus disease 2019 (COVID-19). As of Oct 21, 2020, a total of 41,118,064 confirmed COVID-19 cases and 1,129,710 deaths have been reported worldwide (https://coronavirus.jhu.edu/). The number of patients and death are estimated to increase furthermore. According to the death rates, COVID-19 is the worst pandemic compared with the previous $\mathrm{CoV}$ pandemics- severe acute respiratory syndrome (SARS) and The Middle East respiratory syndrome (MERS) [2,3].

Due to low immunity after chemotherapy, many cancer patients (especially lung cancer patients) frequently have similar pulmonary symptoms as of COVID-19. They easily suffer from fever, either due to infection, drug-induced causes, radiation, chemotherapy-induced febrile neutropenia, or tumor fever. Because of existing abnormalities, they are prone to be infected by COVID-19. Patients who underwent chemotherapy or surgery in the past month possess a much higher risk to get infected [4]. This crisis period raises a lot of challenging questions towards the global oncology community to prioritize the safety considerations for the cancer patients requiring 
essential care in the endemic areas. A set of guidelines and international recommendations are now available for managing cancer patients with special protocols [5].

\section{Coronaviruses that Cause Pandemic}

After a pandemic of severe acute respiratory syndrome (SARS) in 18 years ago and a severe outbreak of Middle East respiratory syndrome (MERS) in 2012 [2, 3], in December 2019, a distinctive coronavirus $(\mathrm{CoV})$ was determined to be responsible for an outbreak of potentially fatal atypical pneumonia, ultimately defined as coronavirus disease 19 (COVID-19), in Wuhan, Hubei province, China. The World Health Organization (WHO) declared the situation pandemic and the biggest threat to public health in the World.

The symptoms and severity of patients from different countries were depicted in Table 1. According to the reports the angiotensin receptor 2 (ACE2) helps the virus to enter the respiratory mucosa [20]. Using the receptor-binding domain of spike protein $\mathrm{S} 1$, the virion attaches to the cell membrane by interacting with host ACE2 receptor in the lower respiratory tracts of infected patients to gain entry into the lungs [21]. This receptor binding capacity is 10 to 20 -fold higher than SARS-CoV and MERS-CoV virus [21]. The receptorbinding domain (RBD) of the S1 subunit then determined the host membrane binding by conformational changes. Figure 1 illustrated the comparison between three different pandemic causing coronaviruses receptor binding structures. It assumes that the receptor binding capacity of SRAS-COV-2 is much higher than SARS-CoV and MERS.

\section{Lifestyle Factors Related to Cancer and SARS-CoV2}

Cancer is a genetic disease that occurred due to genetic alteration of growth-regulatory genes [22]. The major risk factors are lifestyle, which includes diet, age (aging), tobacco and drugs, obesity, alcohol consumption, and infectious diseases [23-26]. Which means the power to minimize and control of cancer is in our own hands. Although some of these cannot be controlled like aging. Human health becomes susceptible with age due to various anatomical, physiological and immunological changes. One of the major changes is our respiratory system, which included chest wall and thoracic spine deformities. These impair the total respiratory system compliance leading to increased work of breathing. With the increasing age, the strength of respiratory muscle decreases. For this reason, older persons are sensible for dyspnea and diminished ventilatory response to hypoxia and hypercapnia. Due to this, they are more vulnerable to ventilatory failure (i.e., heart failure, pneumonia) [27]. Previous studies reported that hospitalization rates for pneumonia range from 1/1000 individuals in the general population to $12 / 1000$ over age 75 years and 33/1000 for residents of chronic care facilities. [28, 29]. In recent pandemic, clinical data showed that COVID-19 and aging have positive correlation and showed a severe high number of COVID-19 related deaths in high age people. (https://www.cdc.gov/mmwr/volumes/69/wr/mm6912e2. htm, https://www.cdc.gov/coronavirus/2019-ncov/needextra-precautions/people-at-higher-risk.html, https://www. cdc.gov/mmwr/volumes/69/wr/mm6915e3.htm, https:// www.latimes.com/science/story/2020-03-31/coronavirusdeath-rate-estimates-show-risk-rising-sharply-with-age, https://www.bloomberg.com/opinion/articles/2020-05-07/ comparing-coronavirus-deaths-by-age-with-flu-drivingfatalities).

Mainstream tobacco smoke (MTS) is the major risk factor in the development and pathogenesis of several lifethreatening diseases [30]. It is estimated that use of tobacco smoke kills around 6 million people annually. About half of all smokers will develop a serious smoking-related illness, such as chronic obstructive pulmonary disease (COPD), which is characterized by irreversible airway obstruction or cardiovascular disease. The most severe adverse effects of cigarette smoke on the immune system, which triggers pathologies such as COPD, respiratory infections, increased incidence and severity of asthma, and cancer are well known nowadays. The immune defects that are caused by smoking relate to the seemingly contradictory nature of smoke as a damaging and pro-inflammatory factor, as well as an immunosuppressive factor. There is lots of evidence, which explores the role of smoking in the risk of lung cancer [31, 32]. According to Food and Drug Administration (FDA) and WHO's statement smoking may increase both the risk of COVID-19 as well as severity; however, no such published data available at this time to establish this correlation. Zhao et al. [33] reported a high correlation between smoking and severity of COVID-19 and show these two are highly significant after analyzing 1726 patients from 7 different studies. In a review with 2986 patients, Emami et al. [34] shows the prevalence of smoking of $7.6 \%$ in the hospitalized patients with COVID-19. Smoking is directly associated with increased risk of virtually every other respiratory infectious disease by destroying airway cilia which is a defense from infectious particles by moving it out of the airway. Additionally, smoking's effect on increasing ACE-2 receptors in the lungs, which is the entry point for the SARS-CoV-2 virus, and theoretically, an increase in receptor density give the virus more points of entry to infect and replicate. (https://www. medpagetoday.com/infectiousdisease/covid19/86144, https:// www.ucsf.edu/news/2020/05/417411/smoking-nearlydoubles-rate-covid-19-progression, https://www.newscientist. com/article/2243944-smoking-probably-puts-you-at-greaterrisk-of-coronavirus-not-less/\#). 


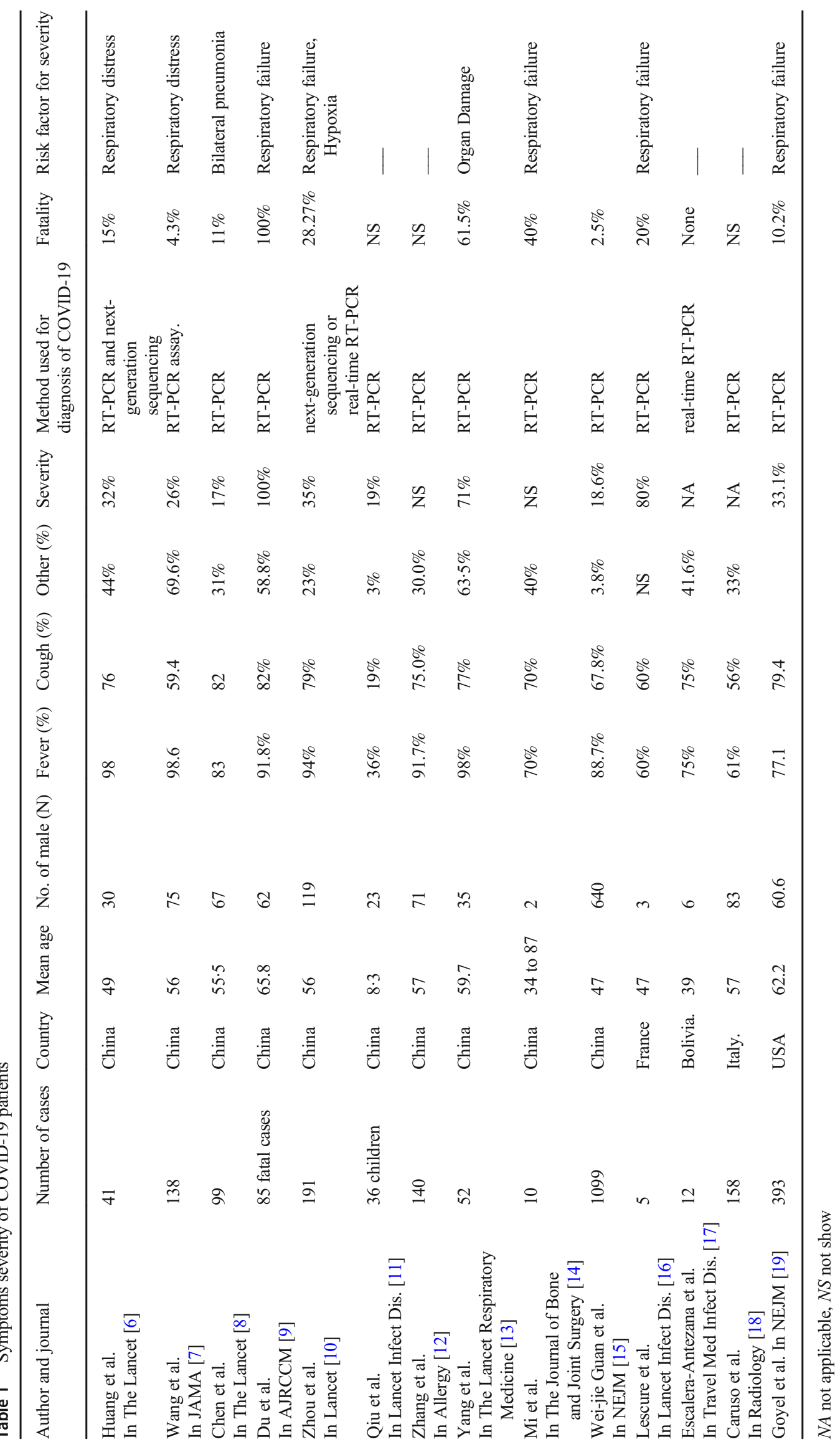


a

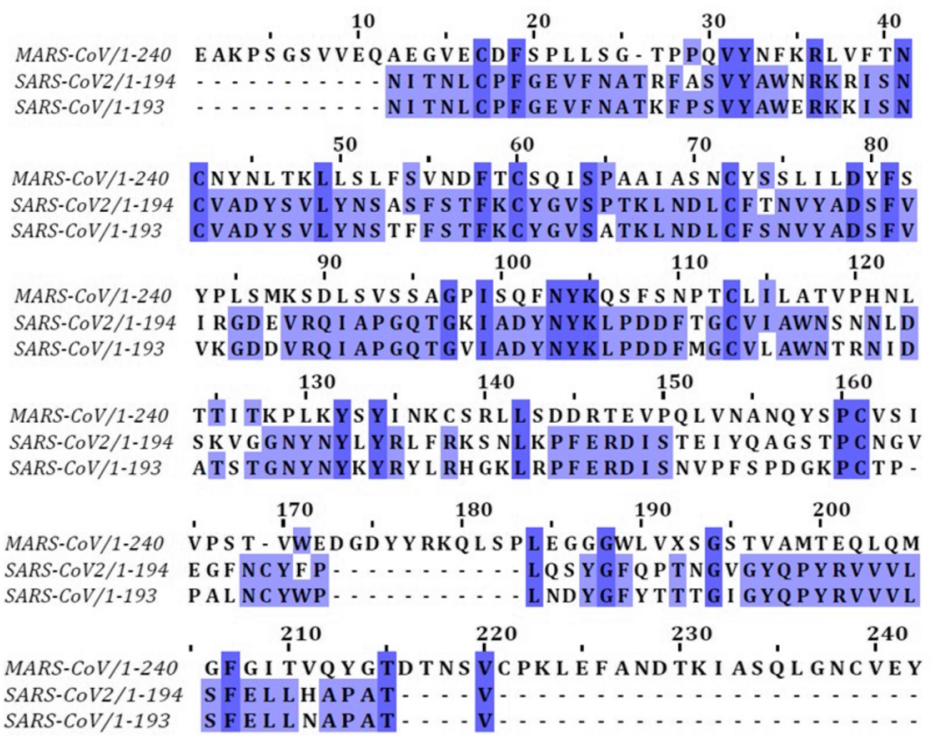

b

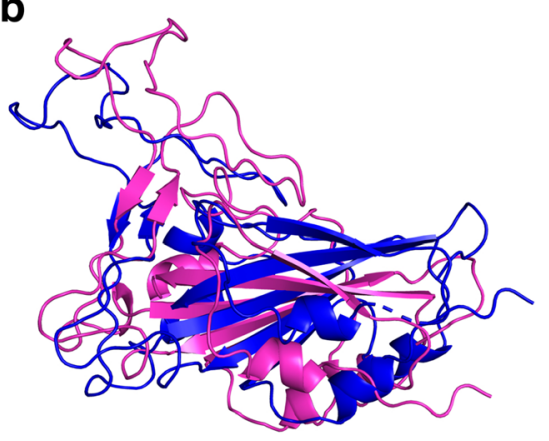

C

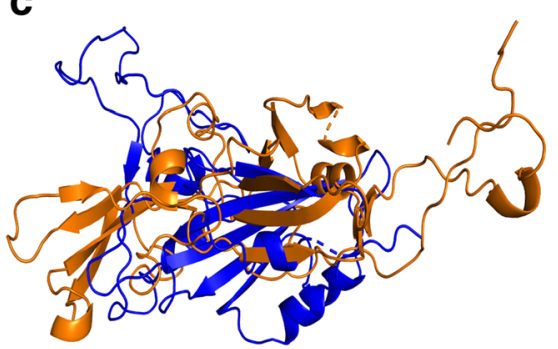

Fig. 1 Characterization of SARS-CoV-2 RBD: a Multiple sequence alignment of RBDs of SARS-CoV-2, SARS-CoV, and MERS-CoV. bc I-tasser online server (https://zhanglab.ccmb.med.umich.edu/ITASSER/) was used to generate a predicted model of the S-protein Receptor binding domain of SARS-CoV2, SARS-CoV, and MERS$\mathrm{CoV}$ to get an idea about the shape of the individual molecules. The

\section{Cancer Patients Are at High Risk of COVID-19}

Patients with cancer are considered as the most vulnerable group in the current COVID-19 pandemic. The increased rate of susceptibility is the cause of their severe systemic immunosuppressive state due to varied anticancer treatment modalities. The alarming widespread nature of the infection raises the concern for its influence on patients with cancer. Thereby both the needs of cancer patients and the workforce caring for them are currently at the forefront of our attention and action. First information on incidence of COVID-19 in cancer patients comes from a study in Wuhan, China, where $0.79 \%$ (12 patients) of the 1524 cancer patients admitted showed infection between 2019 December to 2020 February [35]. Given the rate of contagion was high compared with the collective prevalence in the community $(0.37 \%)$, this report holds limitations in not addressing the COVID-19 incidence amid communityoutpatients with cancer. A recent nationwide analysis in China of 2007 COVID-19 cases declares patients with cancer are at higher risk of severe clinical fatality than those without cancer ( 7 of 18 patients [39\%] vs 124 of 1572 patients [8\%]; Fisher's exact $p=0.0003$ ) [36]. The case fatalness reached $5.6 \%$ amid the cancer cohort compared with $2.3 \%$ of the general population group. Besides, it was reported that the median age of patients with cancer $(63 \cdot 1$ years) is significantly higher than for general population cohort ( $48 \cdot 7$ years). Additional reports in China also suggest hospital-related transmission in both structure of SARS-CoV-2 S (blue) and SARS-CoV S (pink) and MERS-CoV (Orange) RBD domain was superimposed which denotated probable alteration of this binding domain. GenBank accession numbers are QHR63250.2 (SARS-CoV-2 S), ABB29898.2 (SARS-CoV S), and AFS88936.1 (MERS-CoV S)

patients and health care employees. A retrospective case study with 138 patients showed that around $41.3 \%$ have acquired the infection during hospitalization, and of these, 5 patients belong to the oncology section [37]. Some patients with cancer have known to acquire the COVID-19 infection on getting anti-cancer treatment during hospitalization, which supports the susceptibility of cancer patients in the existing pandemic. However, the associated limitation of the study resides on the multiple factors like unavailability of proper clinical information that can correlate the infection risk to the tumor stage among patients. Secondly, the retrospective and nonrandomized form of the study based on a relatively small population size attributes to its limitation. The heterogeneity of the disease among patients and the diverse treatment modalities in practice also contributes to its constraint [38]. Further the report only suggests rudimentary complication and fatality rates of COVID-19 infection in cancer patients; however, comparisons between cancer and non-cancer patients with the infection may possibly reveal more valuable information. Thereby, future studies with large population cohorts and prospective study designs are necessary to reveal the risk issues and severe events in the infected cancer patients.

The outcomes of additional analysis of 105 cancer patients hospitalized for COVID-19 infection over a period of 7 week from 14 hospitals in China were compared with a control group of 536 non-cancer patients and matched for age, hospital, and admission time [39]. Reports demonstrate lung cancer 
$(n=22)$ as the most frequent cancer to be affected in the ongoing pandemic trailed by gastrointestinal (GI, $n=13$ ), thyroid or breast $(n=11)$, and hematological $(n=9)$ cancers. Besides the cancer patients showed high death rates (OR, odds ratio 2.34), increased rates of ICU admission (OR 2.84), higher probability of severe symptoms (OR 2.79), and a two-fold increased susceptibility to having ventilation as compared with the matched non-cancer control patients. Due to limitation of the analysis to be applied to other countries with unlike cancer epidemiology and clinical practice [40], a study was initiated with the investigation of the electronic medical records (EMR) of Mount Sinai Health System (MSHS) in the New York City, USA, to determine whether cancer patients present poor prognosis of COVID-19. A total of 334 patients (6\%) were found with cancer (lung, breast, urothelial, prostate, and colon cancer) among the total 5688 patients with COVID-19 infection [41]. Age-group-dependent stratification of patients declares a significantly increased risk of intubation in cancer patients aged 66 to 80 ; however, patients with cancer younger than age 50 found to have a high mortality rate. This is the first kind of a study in the USA on the prognosis of cancer patients with COVID-19 infection which accounts for its correlation to the patient's age, one of the strongest prognostic factors in determining risk, with a relatively large population cohort. A higher incidence of cancer in COVID-19 infected patients has been stated from New York City, where $6 \%$ of the patients had cancer out of 5700 hospitalized with COVID-19 [42]. Likewise, $8 \%$ of the patients with either present or past history of cancer were reported to be admitted to the intensive care unit for COVID-19 in Lombardy, Italy [43]. Another study from Italy reports $20 \%$ of the deaths from COVID-19 contagion in patients with cancer [44]. Still there arises an urgent need to unravel the logistics behind whether COVID-19-infected cancer patients will have separate clinical courses and whether the patients should receive typical antitumor treatments in the epidemic areas. The current argument emphasizes on whether or not to postpone the anti-cancer treatments, which need to be prepared on a patient-to-patient basis and the associated risk.

\section{Balancing Risks and Benefits in Case of Oncologic Emergencies in the COVID-19 Pandemic}

Given the high susceptibility of the cancer patients in the COVID-19 setting and the associated risk in acquiring the infection during anti-cancer treatments and/or hospitalization, a debate ensues on how to balance the risk factors and benefits out of the oncologic emergencies in cancer patients. Delaying anti-cancer treatment in the ongoing pandemic cannot be recommended as a sensible choice to reduce the associated infection risk in patients. Thus, the health care facilities are required to re-emphasize the integrity and effectiveness of the basic infection control measures to combat the spread of the contagion. The major question lies in how to modify the treatment modalities based on the patient and cancer-specific urgencies. Care of cancer patients generally includes the following discussed forms [45]. First form of care includes the subjects that can be evaluated remotely and are not time-sensitive, which mainly embraces patients who have completed their anti-cancer treatment and possess no acute signs of recurrence or the low-risk patients getting hormonal or oral chemotherapy. Second form of care includes the patients where the treatment delayed or omitted will have a minimal effect on the quality and/or quantity of life. In cases of patients with advanced cancer at times oncology care involves systemic chemotherapy and, in cases where it can be determined that the survival benefit of the chemotherapy is limited, a decision on delay can be taken depending on the potential benefit of therapy vs the risk if the patient becomes contagious. Likewise, delaying surgery and directing neoadjuvant therapy for the moment can reduce risk to the patient and reserve the health care resources. A report on the measures taken at the cancer center in New York, USA, includes a comprehensive departmental strategy to triage and shorten the radiation therapy for oncologic crises. According to the report, the oncology department has logically implemented a three-tiered system in the ongoing pandemic to characterize clinically urgent cases, where postponing therapy would result in compromised outcomes or severe morbidity [46]. Tier-1 care includes patients with metastatic cancer requiring palliative radiotherapy as urgent need. Tier-2 care embraces patients with symptomatic disease lacking oncologic crises in which radiotherapy is the basic standard of care, and patients with asymptomatic disease in which radiotherapy is suggested to avert imminent functional deficits. Tier-3 care includes patients with asymptomatic or symptomatic disease in which radiotherapy functions as one of the effective treatment strategies. Management in the described manner may help the radiation oncologists to exclude, delay, or impose short-term courses to lessen the number of hospital visits in the setting of COVID-19 pandemic. Third form of care includes the patients where delay in treatment has an adverse impact on the survival or quality of life. In most cases, for several cancer types, the oncologists are advising marginally less effective drug regimens that have minor risk of causing hospitalization [45]. Current practice recommends the oncologists to reasonably use stringent neutrophil counts and the white cell growth factor before ensuing with the next therapy cycle, and restricting usage of steroids to manage nausea. Whenever feasible, oral therapies are replaced with intravenous form and different other modifications are taken into account to lessen the patient visits and/or hospitalizations. To further prioritize the situation therapy schemes were modified and the treatments expected to cause immunosuppression should be avoided or low dosages to be provided, 
whereas patients in poor health condition will not receive such treatments. Likely, at least a week ahead before anti-cancer treatment, the cancer patients should stay under observation of the consultants and in isolation. Additionally, strong personal protection including their families must be prepared for the patients. Fourth form of care comprises the patients where the treatment owns the potential to cure and cannot safely be postponed, which includes new diagnoses of high-grade lymphoma, acute leukemia, and the therapy-responsive cancers small cell lung, ovarian, and testicular cancers [45]. Depending on the lethality associated with these cancers, the oncologists are not modifying the therapy regimens despite the COVID-19 risk. For most allogeneic transplants, which cannot be safely delayed, both the recipients and the donors were introduced to the COVID-19 testing before the start of induction.

Ensuring the risk associated with cancer patient's care and the risk of contracting COVID-19 infection the subsequent step is to consider the ability of the regional health care organization to meet the projected and prevailing needs. To this awareness is required to understand whether the organization is in the preparative, acute, or crisis stage of the pandemic. The preparative stage discloses the integrity of the health care workforce and ensures the availability of the necessary equipment including ventilators, intensive care units (ICUs), and the patient beds [47]. The acute stage deals with when there is limited workforce in the system but still functional through strategic reformation to provide the basic cancer care to their patients. The crisis stage deals with when the system over surpluses with the COVID-19 cases and deprived of its ability to provide cancer patient care.

\section{Strategic Maintenance of Patients with Cancer Having the COVID-19 Testing: Positive for the Infection}

Overall, immunosuppressive therapy in cancer was decided to be suspended in patients who test positive for the infection. A case study reports that ibrutinib (Bruton's tyrosine kinase inhibitor) for chronic hematologic malignant patients might be an exception [48] and the treatment should be considered on a particular case basis [49]. Moreover, decision-making for cancer patients getting glucocorticoids diagnosed with COVID19 must be personalized and put forward on a case-by-case basis. However, non-immunosuppressive oral treatments including hormonal treatments or therapies targeting the activating mutations (like EGFR/MEK inhibitors) must be individualized. Cancer patients at an advanced stage or with comorbid health disorders affecting the lungs or the heart, if they acquire COVID-19 infection, the diagnosis is expected to be dismal [50]. Medical decisions depend on the practical discussions of the consultants with their patients on the goals of advance care planning [51]. Several approaches were prioritized by critical oncology organizations in the ongoing pandemic setting. The American Society of Clinical Oncology (ASCO) recommends that in cancer patients diagnosed with the infection, the immunosuppressive therapies should be withheld until the symptoms resolve like complete remission of fever without use of antipyretics along with a negative COVID-19 test. Furthermore, the US Centers for Disease Control and Prevention (CDC) includes more stringent guidelines on calling of a negative test result using the FDA-approved molecular assay technique for COVID-19 from at least two successive negative nasopharyngeal swab specimens that are collected $24 \mathrm{~h}$ apart. Likewise, the World Health Organization (WHO) suggests that the patients be tested negative using two consecutive specimens collected at 24-h interval before release from home quarantine. On the contrary, the UK National Institute for Health and Care Excellence (NICE) declared published guidelines on initiating or resuming the anticancer therapy only after a single negative COVID-19 test. Some institutions also recommend sending the cancer patients recovered from COVID-19 infection to resume therapy at an isolated infusion center distant from the core infusion center depending on the available resources [52]. Nevertheless, contact limitation and physical distancing guidelines continue to be an important part of the cancer treatment strategies during the pandemic in order to protect the patients, health-care personnel and non-COVID-19 patients being treated in the same organization.

\section{Ensuring Care During the Pandemic}

The ASCO prioritize several guidelines provided by the CDC in the current pandemic setting. Firstly, the patients should be made aware of COVID-19 symptoms and should be trained in executing accurate hand wash, maintaining hygiene and minimizing exposure to infected contacts and huge gatherings. Both patients and health workers need to follow the CDC guidelines on mask use whenever out. As already discussed, any clinic visit that can be delayed without causing any risk to the patient should be suspended. A pre-screening strategy through digital platforms or phone calls for COVID-19 symptoms and acquaintance history preceding planned health center visits in-person is recommended [39]. Additional precautions include proper maintenance of hygiene and cleanliness of the health care centers and the workforces cleaning rooms must use full PPE if the patients were suspected or confirmed of having the contagion. Besides, the pandemic demands adequate availability and use of personal protective equipment (PPE) as per the CDC (https://www.cdc.gov/coronavirus/ 2019-ncov/healthcare-facilities/guidance-hcf.html) and the American College of Surgeons (ACS) (https://www.facs.org/ covid-19/clinical-guidance) guidelines to protect all health 
workforces from being infected. Further to optimize protection to the health personnel from infection, which spreads with an aerosol transmission through droplets and surfaces [53], planning a negative pressure operating room (OR) and anteroom was recommended by the ACS (https:// www.facs.org/covid-19/clinical-guidance) and by Liang et al. (https://www.alnap.org/help-library/handbook-of-covid-19prevention-and-treatment) for COVID-19 suspected or positive patients. For a negative pressure OR, due to the low air pressure created inside than its surroundings, the air that flows into the room cannot escape, thereby protecting workforces in the same center. Particularly, extubation, a risky process comprising viral spread is recommended to be executed either in the negative pressure or in a committed anesthesia recovery room [54]. Additional consideration is the course of surgical access where minimal invasive surgery with pneumoperitoneum, an aerosol generating procedure (AGP) must be cautiously considered. Reports suggest active replication of COVID19 virus in the respiratory and GI tracts [55, 56], thus during the digestive surgeries, the probable risk for aerosol transmission has been warned and a passive evacuation system to be used with electrostatic filter from ventilators has been proposed [57] to protect health care personnel. Likely, the readiness of a plethora of guidelines possibly will be helpful and approachable given the discrepancies in resources available round the world.

\section{Oncology Community United in the COVID-19 Setting}

Management of cancer in recent days are subjected to clinical research trials, which are an integrated part of the routine cancer care, providing additional treatment opportunities for eligible patients. A critical barrier has been imposed to the enrollment and ongoing participation in clinical trials due to the COVID-19 pandemic [58]. The international bodies including the US FDA and the European Medicines Agency have stated proper guidelines for the sponsors and study centers to warrant the safety of participation in trial studies, through reducing the risks to the study integrity and maintenance of other regulatory compliances [59]. Though the clinical trial accretion has reduced severely along with the availability of bio-specimens for various correlative analyses, the oncologists still focus on the therapeutic trial participants so that they can receive their protocol directed treatment and assessments wherever it is safe. With the continuation of the crisis period, the oncologists report unparalleled levels of companionship and cooperation to share responsibility across the world. The oncology community across the world was reported to be united to share therapy schemes, to formulate the best possible guidelines to help cancer patients, to accumulate and assemble the available data, and to strategize and execute therapy/trial protocols. Several associations came up unitedly with identification of patients at the risk of being infected and determining how the anti-cancer treatment influences the existing COVID-19 illness in them and critically analyze whether it is safe to resume the treatment in the contagious patients. The critical question remains on how to maintain the clinical standards so that the value of cancer treatments with the challenging risks during this period of declining resources will meet the expectations of humanitarian welfare. Since the present situation deals with prohibition of visitors accompanying patients, the oncology clinicians and cancer patients must pay attention on clarifying progress directives, prioritize health care substitutes and end-of-life care preferences [51]. Several guidelines from a group of experts across the world were dedicated to provide care to certain cancer types that can be affected mostly by COVID-19 infection. For breast cancer, the European Society of Medical Oncology (ESMO) and the American Society of Breast Surgeons have issued guidelines on providing interdisciplinary care in breast cancer patients and another international group has prioritized guidance for radiation therapies in early breast cancer cases [60]. Besides the guidelines on colorectal surgery from the American College of Surgeons (ACS) and GI-tract cancer surgery from the ESMO, guidance to minimize risk in GI malignant patients has been also developed by an American group of oncologists [61] and the US Colorectal Cancer Alliance. Likewise the management of gynecologic cancers is further developed by the Society of Gynecologic Oncology, the International Journal of Gynecologic Cancer [62], the National College of French Gynecologists and obstetricians [63], and from the International Gynecologic Cancer Society, in addition to that of the ACS and the ESMO. An important consideration in the ongoing pandemic is the patients with lung cancer. The Thoracic Surgery Outcomes Research Network has put forward the guidelines on triage for thoracic surgery in the thoracic cancer patients [64] in addition to that of the ACS. Besides ESMO published specific strategies on the care of different lung cancer types that should be considered with high, medium and low priority depending on the associated risk and the stage of the cancer patients with the available resources. Additional to the basic guidance from the American Society of Transplantation and Cellular Therapy (ASTCT), the American Society of Hematology, the ESMO, and the European Society for Blood and Marrow Transplantation (EBMT) guidelines on the management of patients with hematologic malignancies was also published by the Seattle Cancer Care Alliance [65]. Further improvised radiation therapy strategies were provided by the International Lymphoma Radiation Oncology Group [66]. Besides an international group put forward the care of aged multiple myeloma patients [67], whereas the US Cutaneous Lymphoma consortium guides therapy of the cutaneous lymphoma [68]. 
For the maintenance of hepatocellular cancer in addition to the ESMO, guidelines were also established by the International Liver Cancer Association, the French Association for the Study of the Liver [69] and the American Association for the Study of Liver Diseases (AASLD) [70]. In case of head and neck cancer the specific triage recommendations for surgery in COVID-19 pandemic was provided by the University of Texas MD Anderson Cancer Center [71] and an expert group comprising of the French Society of Otolaryngology, the Head and Neck Surgery and the French Society of Head and Neck Carcinology [72]. Care of patients with brain tumors has been established by the American Association of Neurological Surgeons, the Society for Neuro-Oncology, and the Congress of Neurological Surgeons Tumor Section [73], and detailed guidelines for care of the glioma patients have been obtained from an international multidisciplinary group [74]. Beyond this, a recent article in Morocco that report on the possible ways to minimize the risk of spreading infection in cancer patients in the post-COVID-19 period explains the need to strategize and implement a national screening awareness for the infection to all the patients in order to protect them and the medical supervisors from the disease [75]. Recommendations for the management of COVID-19 negative patients should be prioritize depending on the urgency of their health condition, while a dedicated surveillance and optimal care should be implicated for all the positive individuals where the critical management cannot be postponed in the post-COVID-19 period. Every crisis period brings with it the power to create several new opportunities in order to develop and expand our professional knowledge, available techniques, and accurate exploitation of the reduced resources and perhaps most importantly the selfurge to unite ourselves and show the unparalleled levels of companionship as a large health care community towards serving for the benefit of our patients.

\section{Conclusion}

The outbreak of COVID-19 has caused pandemic worldwide, which is much more effective than SARS and MERS, which are experienced by the world. Due to lack of knowledge and work, there is no such evidence that proves the possible treatment approach of cancer patients. It is hard to make management decisions due to lack of data. At this time, the most challenging part is delaying cancer treatment, especially patients with COVID-19 infection. In our belief, the clinicians should take all the care and report proper case reports of COVID-19 in patients with malignant co-morbidities. More detailed etiologic and risk factor study is essential in case to cancer patients during this pandemic situation.

\section{Compliance with Ethical Standards}

Conflict of Interest The authors declare that they have no conflict of interest.

\section{References}

1. Zhu N, Zhang D, Wang W, Li X, Yang B, Song J, et al. A novel coronavirus from patients with pneumonia in China, 2019. N Engl J Med. 2020;382:727-33.

2. Drosten C, Gunther S, Preiser W, van der Werf S, Brodt HR, Becker $\mathrm{S}$, et al. Identification of a novel coronavirus in patients with severe acute respiratory syndrome. N Engl J Med. 2003;348:196776.

3. Zaki AM, van Boheemen S, Bestebroer TM, Osterhaus AD, Fouchier RA. Isolation of a novel coronavirus from a man with pneumonia in Saudi Arabia. N Engl J Med. 2012;367:1814-20.

4. Al-Shamsi HO, Alhazzani W, Alhuraiji A, Coomes EA, Chemaly $\mathrm{RF}$, Almuhanna M, et al. A practical approach to the management of cancer patients during the novel coronavirus disease 2019 (COVID-19) pandemic: an international collaborative group. Oncologist. 2020;25:e936-45.

5. Shankar A, Saini D, Roy S, Mosavi Jarrahi A, Chakraborty A, Bharti SJ, et al. Cancer care delivery challenges amidst coronavirus disease - 19 (COVID-19) outbreak: specific precautions for cancer patients and cancer care providers to prevent spread. Asian Pac J Cancer Prev. 2020;21:569-73.

6. Huang C, Wang Y, Li X, Ren L, Zhao J, Hu Y, et al. Clinical features of patients infected with 2019 novel coronavirus in Wuhan, China. Lancet. 2020;395:497-506.

7. Wang D, Hu B, Hu C, Zhu F, Liu X, Zhang J, et al. Characteristics of 138 hospitalized patients with 2019 novel coronavirus-infected pneumonia in Wuhan, China. JAMA. 2020;323:1061-9.

8. Chen N, Zhou M, Dong X, Qu J, Gong F, Han Y, et al. Epidemiological and clinical characteristics of 99 cases of 2019 novel coronavirus pneumonia in Wuhan, China: a descriptive study. Lancet. 2020;395:507-13.

9. Du Y, Tu L, Zhu P, Mu M, Wang R, Yang P, et al. Clinical features of 85 fatal cases of COVID-19 from Wuhan. A retrospective observational study. Am J Respir Crit Care Med. 2020;201:1372-9.

10. Zhou F, Yu T, Du R, Fan G, Liu Y, Liu Z, et al. Clinical course and risk factors for mortality of adult inpatients with COVID-19 in Wuhan, China: a retrospective cohort study. Lancet. 2020;395: 1054-62.

11. Qiu H, Wu J, Hong L, Luo Y, Song Q, Chen D. Clinical and epidemiological features of 36 children with coronavirus disease 2019 (COVID-19) in Zhejiang, China: an observational cohort study. Lancet Infect Dis. 2020;20:689-96.

12. Zhang JJ, Dong X, Cao YY, Yuan YD, Yang YB, Yan YQ, et al. Clinical characteristics of 140 patients infected with SARS-CoV-2 in Wuhan. China Allergy. 2020;75:1730-41.

13. Yang X, Yu Y, Xu J, Shu H, Xia J, Liu H, et al. Clinical course and outcomes of critically ill patients with SARS-CoV-2 pneumonia in Wuhan, China: a single-centered, retrospective, observational study. Lancet Respir Med. 2020;8:475-81.

14. Mi B, Chen L, Xiong Y, Xue H, Zhou W, Liu G. Characteristics and early prognosis of COVID-19 infection in fracture patients. $\mathrm{J}$ Bone Joint Surg Am. 2020;102:750-8.

15. Guan WJ, Ni ZY, Hu Y, Liang WH, Ou CQ, He JX, et al. Clinical characteristics of coronavirus disease 2019 in China. N Engl J Med. 2020;382:1708-20.

16. Lescure FX, Bouadma L, Nguyen D, Parisey M, Wicky PH, Behillil S, et al. Clinical and virological data of the first cases of 
COVID-19 in Europe: a case series. Lancet Infect Dis. 2020;20: 697-706.

17. Escalera-Antezana JP, Lizon-Ferrufino NF, Maldonado-Alanoca A, Alarcón-De-la-Vega G, Alvarado-Arnez LE, BalderramaSaavedra MA, et al. Clinical features of the first cases and a cluster of Coronavirus Disease 2019 (COVID-19) in Bolivia imported from Italy and Spain. Travel Med Infect Dis. 2020;35:101653.

18. Caruso D, Zerunian M, Polici M, Pucciarelli F, Polidori T, Rucci C, et al. Chest CT Features of COVID-19 in Rome. Italy Radiology. 2020;296:E79-85.

19. Goyal P, Choi JJ, Pinheiro LC, Schenck EJ, Chen R, Jabri A, et al. Clinical characteristics of Covid-19 in New York City. N Engl J Med. 2020;382:2372-4.

20. Cheng ZJ, Shan J. 2019 Novel coronavirus: where we are and what we know. Infection. 2020;48:155-63.

21. Wrapp D, Wang N, Corbett KS, Goldsmith JA, Hsieh CL, Abiona O, et al. Cryo-EM structure of the 2019-nCoV spike in the prefusion conformation. Science. 2020;367:1260-3.

22. Futreal PA, Coin L, Marshall M, Down T, Hubbard T, Wooster R, et al. A census of human cancer genes. Nat Rev Cancer. 2004;4: 177-83.

23. Khan N, Afaq F, Mukhtar H. Cancer chemoprevention through dietary antioxidants: progress and promise. Antioxid Redox Signal. 2008; 10:475-510

24. Surh YJ. Cancer chemoprevention with dietary phytochemicals. Nat Rev Cancer. 2003;3:768-80.

25. Khan N, Adhami VM, Mukhtar H. Apoptosis by dietary agents for prevention and treatment of cancer. Biochem Pharmacol. 2008;76: 1333-9.

26. Khan N, Afaq F, Mukhtar H. Lifestyle as risk factor for cancer: evidence from human studies. Cancer Lett. 2010;293:133-43.

27. Sharma G, Goodwin J. Effect of aging on respiratory system physiology and immunology. Clin Interv Aging. 2006;1:253-60.

28. Fein AM. Pneumonia in the elderly: overview of diagnostic and therapeutic approaches. Clin Infect Dis. 1999;28:726-9.

29. Meyer KC. Lung infections and aging. Ageing Res Rev. 2004;3: 55-67.

30. Bhat TA, Kalathil SG, Bogner PN, Miller A, Lehmann PV, Thatcher TH, et al. Secondhand smoke induces inflammation and impairs immunity to respiratory infections. J Immunol. 2018;200: 2927-40.

31. Chen R, Lin J. Identification of feature risk pathways of smokinginduced lung cancer based on SVM. PLoS One. 2020;15: e0233445.

32. Yang Z, Zhuan B, Yan Y, Jiang S, Wang T. Identification of gene markers in the development of smoking-induced lung cancer. Gene. 2016;576:451-7.

33. Zhao Q, Meng M, Kumar R, Wu Y, Huang J, Lian N, et al. The impact of COPD and smoking history on the severity of COVID19: A systemic review and meta-analysis. J Med Virol. 2020. https://doi.org/10.1002/jmv.25889.

34. Emami A, Javanmardi F, Pirbonyeh N, Akbari A. Prevalence of underlying diseases in hospitalized patients with COVID-19: a systematic review and meta-analysis. Arch Acad Emerg Med. 2020;8:e35.

35. Yu J, Ouyang W, Chua MLK, Xie C. SARS-CoV-2 Transmission in patients with cancer at a tertiary care hospital in Wuhan. China JAMA Oncol. 2020;6:1108-10.

36. Liang W, Guan W, Chen R, Wang W, Li J, Xu K, et al. Cancer patients in SARS-CoV-2 infection: a nationwide analysis in China. Lancet Oncol. 2020;21:335-7.

37. Zhang L, Zhu F, Xie L, Wang C, Wang J, Chen R, et al. Clinical characteristics of COVID-19-infected cancer patients: a retrospective case study in three hospitals within Wuhan. China Ann Oncol. 2020;31:894-901.

38. Xia Y, Jin R, Zhao J, Li W, Shen H. Risk of COVID-19 for patients with cancer. Lancet Oncol. 2020;21:e180.
39. Dai M, Liu D, Liu M, Zhou F, Li G, Chen Z, et al. Patients with cancer appear more vulnerable to SARS-CoV-2: A multicenter study during the COVID-19 outbreak. Cancer Discov. 2020;10: 783-91.

40. Oh WK. COVID-19 infection in cancer patients: early observations and unanswered questions. Ann Oncol. 2020;31:838-9.

41. Miyashita H, Mikami T, Chopra N, Yamada T, Chernyavsky S, Rizk D, et al. Do patients with cancer have a poorer prognosis of COVID-19? An experience in New York City. Ann Oncol. 2020;31:1088-9.

42. Richardson S, Hirsch JS, Narasimhan M, Crawford JM, McGinn T, Davidson KW, et al. Presenting characteristics, comorbidities, and outcomes among 5700 patients hospitalized with COVID-19 in the New York City area. JAMA. 2020;323:2052-9.

43. Grasselli G, Zangrillo A, Zanella A, Antonelli M, Cabrini L, Castelli A, et al. Baseline characteristics and outcomes of 1591 patients infected with SARS-CoV-2 admitted to icus of the lombardy region. Italy JAMA. 2020;323:1574-81.

44. Onder G, Rezza G, Brusaferro S. Case-fatality rate and characteristics of patients dying in relation to COVID-19 in Italy. JAMA. 2020;323:1775-6.

45. Schrag D, Hershman DL, Basch E. Oncology practice during the COVID-19 pandemic. JAMA. 2020;323:2005-6.

46. Yerramilli D, Xu AJ, Gillespie EF, Shepherd AF, Beal K, Gomez $\mathrm{D}$, et al. Palliative radiotherapy for oncologic emergencies in the setting of COVID-19: approaches to balancing risks and benefits. Adv Radiat Oncol. 2020;5:589-94.

47. Cinar P, Kubal T, Freifeld A, Mishra A, Shulman L, Bachman J, et al. Safety at the time of the COVID-19 pandemic: How to keep our oncology patients and healthcare workers safe. J Natl Compr Canc Netw. 2020;1-6. https://doi.org/10.6004/jnccn.2020.7572.

48. Treon SP, Castillo JJ, Skarbnik AP, Soumerai JD, Ghobrial IM, Guerrera ML, et al. The BTK inhibitor ibrutinib may protect against pulmonary injury in COVID-19-infected patients. Blood. 2020;135:1912-5.

49. Chong EA, Roeker LE, Shadman M, Davids MS, Schuster SJ, Mato AR. BTK inhibitors in Cancer patients with COVID-19: "the winner will be the one who controls that Chaos" (Napoleon Bonaparte). Clin Cancer Res. 2020;26:3514-6.

50. Ueda M, Martins R, Hendrie PC, McDonnell T, Crews JR, Wong TL, et al. Managing cancer care during the COVID-19 Pandemic: Agility and collaboration toward a common goal. J Natl Compr Canc Netw. 2020;1-4. https://doi.org/10.6004/jnccn.2020.7560.

51. Curtis JR, Kross EK, Stapleton RD. The importance of addressing advance care planning and decisions about do-not-resuscitate orders during novel coronavirus 2019 (COVID-19). JAMA. 2020;323:1771-2.

52. Lan L, Xu D, Ye G, Xia C, Wang S, Li Y, et al. Positive RT-PCR test results in patients recovered from COVID-19. JAMA. 2020;323:1502-3.

53. Li Q, Guan X, Wu P, Wang X, Zhou L, Tong Y, et al. Early transmission dynamics in Wuhan, China, of novel coronavirusinfected pneumonia. N Engl J Med. 2020;382:1199-207.

54. Ti LK, Ang LS, Foong TW, Ng BSW. What we do when a COVID-19 patient needs an operation: operating room preparation and guidance. Can J Anaesth. 2020;67:756-8.

55. Wolfel R, Corman VM, Guggemos W, Seilmaier M, Zange S, Muller MA, et al. Virological assessment of hospitalized patients with COVID-2019. Nature. 2020;581:465-9.

56. Xiao F, Tang M, Zheng X, Liu Y, Li X, Shan H. Evidence for gastrointestinal infection of SARS-CoV-2. Gastroenterology. 2020;158:1831-3 e3.

57. Mintz Y, Arezzo A, Boni L, Chand M, Brodi R, Fingerhut A, et al. Response to letter to the editor about our article "A low cost, safe and effective method for smoke evacuation in laparoscopic surgery 
for suspected coronavirus patients." Ann Surg. 2020. https://doi. org/10.1097/SLA.0000000000004215.

58. Waterhouse DM, Harvey RD, Hurley P, Levit LA, Kim ES, Klepin HD, et al. Early Impact of COVID-19 on the Conduct of Oncology Clinical Trials and Long-Term Opportunities for Transformation: Findings From an American Society of Clinical Oncology Survey. JCO Oncol Pract. 2020;16:417-21.

59. de Paula BHR, Araujo I, Bandeira L, Barreto N, Doherty GJ. Recommendations from national regulatory agencies for ongoing cancer trials during the COVID-19 pandemic. Lancet Oncol. 2020;21:624-7.

60. Coles CE, Aristei C, Bliss J, Boersma L, Brunt AM, Chatterjee S, et al. International guidelines on radiation therapy for breast cancer during the COVID-19 pandemic. Clin Oncol (R Coll Radiol). 2020;32:279-81.

61. Lou E, Beg S, Bergsland E, Eng C, Khorana A, Kopetz S, et al. Modifying Practices in GI Oncology in the Face of COVID-19: Recommendations From Expert Oncologists on Minimizing Patient Risk. JCO Oncol Pract. 2020;16:383-8.

62. Ramirez PT, Chiva L, Eriksson AGZ, Frumovitz M, Fagotti A, Gonzalez Martin A, et al. COVID-19 global pandemic: options for management of gynecologic cancers. Int $\mathrm{J}$ Gynecol Cancer. 2020;30:561-3.

63. Akladios C, Azais H, Ballester M, Bendifallah S, Bolze PA, Bourdel N, et al. Recommendations for the surgical management of gynecological cancers during the COVID-19 pandemicFRANCOGYN group for the CNGOF. J Gynecol Obstet Hum Reprod. 2020;49:101729.

64. Thoracic Surgery Outcomes Research Network, Inc, Antonoff M, Backhus L, Boffa DJ, Broderick SR, Brown LM, et al. COVID-19 guidance for triage of operations for thoracic malignancies: A consensus statement from Thoracic Surgery Outcomes Research Network. J Thorac Cardiovasc Surg. 2020;160:601-605.

65. Percival MM, Lynch RC, Halpern AB, Shadman M, Cassaday RD, Ujjani C, et al. Considerations for Managing Patients With Hematologic Malignancy During the COVID-19 Pandemic: The Seattle Strategy. JCO Oncol Pract. 2020;16:571-8.

66. Yahalom J, Dabaja BS, Ricardi U, Ng A, Mikhaeel NG, Vogelius IR, et al. ILROG emergency guidelines for radiation therapy of hematological malignancies during the COVID-19 pandemic. Blood. 2020;135:1829-32.

67. Mian H, Grant SJ, Engelhardt M, Pawlyn C, Bringhen S, Zweegman S, et al. Caring for older adults with multiple myeloma during the COVID-19 pandemic: perspective from the international forum for optimizing care of older adults with myeloma. J Geriatr Oncol. 2020;11:764-8.

68. Zic JA, Ai W, Akilov OE, Carter JB, Duvic M, Foss F, et al. United States cutaneous lymphoma consortium recommendations for treatment of cutaneous lymphomas during the COVID-19 pandemic. J Am Acad Dermatol. 2020;83:703-4.

69. Ganne-Carrie N, Fontaine H, Dumortier J, Boursier J, Bureau C, Leroy $\mathrm{V}$, et al. Suggestions for the care of patients with liver disease during the coronavirus 2019 pandemic. Clin Res Hepatol Gastroenterol. 2020;44:275-81.

70. Fix OK, Hameed B, Fontana RJ, Kwok RM, McGuire BM, Mulligan DC, et al. Clinical best practice advice for hepatology and liver transplant providers during the COVID-19 pandemic: AASLD expert panel consensus statement. Hepatology. 2020;72: 287-304.

71. Head MDA, Neck surgery treatment guidelines C, consortium m, Maniakas A, Jozaghi Y, Zafereo ME, et al. Head and neck surgical oncology in the time of a pandemic: subsite-specific triage guidelines during the COVID-19 pandemic. Head Neck. 2020;42:1194 201.

72. Fakhry N, Schultz P, Moriniere S, Breuskin I, Bozec A, Vergez S, et al. French consensus on management of head and neck cancer surgery during COVID-19 pandemic. Eur Ann Otorhinolaryngol Head Neck Dis. 2020;137:159-60.

73. Ramakrishna R, Zadeh G, Sheehan JP, Aghi MK. Inpatient and outpatient case prioritization for patients with neuro-oncologic disease amid the COVID-19 pandemic: general guidance for neurooncology practitioners from the AANS/CNS Tumor Section and Society for Neuro-Oncology. J Neuro-Oncol. 2020;147:525-9.

74. Mohile NA, Blakeley JO, Gatson NTN, Hottinger AF, Lassman $\mathrm{AB}, \mathrm{Ney} \mathrm{DE}$, et al. Urgent considerations for the neuro-oncologic treatment of patients with gliomas during the COVID-19 pandemic. Neuro-Oncology. 2020;22:912-7.

75. Ismaili N. COVID-19 Recommendations for patients with cancer: The post-COVID-19 Era. SN Compr Clin Med. 2020;1-6. https:// doi.org/10.1007/s42399-020-00425-7.

Publisher's Note Springer Nature remains neutral with regard to jurisdictional claims in published maps and institutional affiliations. 